\title{
THE HIGGS SECTOR AND ELECTRON ELECTRIC DIPOLE MOMENT IN NEXT-TO-MINIMAL SUPERSYMMETRY WITH EXPLICIT CP VIOLATION
}

\author{
MÜGE BOZ \\ Physics Department, Hacettepe University \\ Ankara, 06532, Turkey
}

\begin{abstract}
We study the explicit CP violation of the Higgs sector in the next-to-minimal supersymmetric model with a gauge singlet Higgs field. Our numerical predictions show that electric dipole moment of electron lies around the present experimental upper limits. The mass of the lightest Higgs boson is quite sensitive to the $\mathrm{CP}$ violating phases in the theory. It is observed that as the vacuum expectation value of the singlet gets higher values, $\mathrm{CP}$ violation increases.
\end{abstract}

\section{Introduction}

The present bounds on the electric dipole moments (EDM) of the particles generate serious hierarchy problems concerning the amount of $\mathrm{CP}$ violation. For instance, the neutron EDM ${ }^{1}$, induced by the the $\mathrm{QCD}$ vacuum angle $\left(\theta_{Q C D}\right)$, is approximately ten orders of magnitude larger than the existing bound, which is the source of the so-called strong CP problem. This naturalness problem has been solved by the Peccei-Quinn mechanism ${ }^{2}$ which promotes $\theta_{Q C D}$ to a dynamical variable via the phases of the quarks or additional color triplets ${ }^{2,3,4}$.

In the supersymmetric (SUSY) extensions of the standard electroweak theory (SM) this hierarchy problem still persists. Moreover, there appear novel sources of $\mathrm{CP}$ violation coming from the soft supersymmetry breaking mass terms. Though the phases of the soft terms have been shown to relax to $\mathrm{CP}$-conserving points in the minimal model (MSSM) ${ }^{5}$, this is not necessarily true in the non-minimal model (NMSSM) ${ }^{6}$ containing a singlet. The Lagrangian of the MSSM consists of various mass parameters which are not necassarily real ${ }^{7}$. The phases of these parameters contribute to known $\mathrm{CP}$ violation observables such as the electric dipole moments of the electron, neutron, atoms, and molecules ${ }^{8,9,10,11,12,13}$, the Higgs system ${ }^{14,15,16}$, and the decays and mixings of mesons ${ }^{17}$.

In addition to these CP hierarchy problems, in minimal SUSY model there is another hierarchy problem concerning the Higgsino Dirac mass parameter $(\mu)$, that is, this mass parameter follows from the superpotential of the model and there is no telling of at what scale (ranging from $M_{W}$ to $M_{P l}$ ) it is stabilized.

The next-to-minimal supersymmetric model (NMSSM) is the most economic extension of the MSSM in which the $\mu$ parameter is induced by the vacuum expectation value (VEV) of an additional gauge singlet. The NMSSM not only solves the $\mu$ problem by means of the VEV of the singlet, but also offers a rich phenomenology for colliders ${ }^{18}$. 
In the NMSSM, the Higgs sector of the MSSM (including two CP-even Higgs bosons, a CP odd Higgs boson, and a charged Higgs pair) is extended so that there are three $\mathrm{CP}$-even Higgs bosons, two CP-odd Higgs bosons, and a charged Higgs pair. Therefore, the Higgs phenomenology of the NMSSM significantly differ from that of MSSM ${ }^{19,20}$. As a result of the SM searches at LEP, the lower bound on the lightest Higgs boson mass is $115 \mathrm{GeV}$ (and correspondingly $\tan \beta \gtrsim 3.5{ }^{21}$. Therefore, from the searches at LEP2, the lower limit on the mass of the SM Higgs boson excludes the substantial part of the MSSM parameter space particularly (for $\left.m_{t}=175 \mathrm{GeV}\right)$ at low $\tan \beta(\tan \beta \lesssim 3.5)$. However, in the low $\tan \beta$ regime, NMSSM is in much better shape phenomenologically, since the Higgs boson masses are larger, and the fine-tuning is less ${ }^{22,23}$. Thus, the phenomenological implications of the NMSSM can offer new opportunities at future colliders ${ }^{19}$.

In the MSSM, since the tree level vacua are $\mathrm{CP}$ conserving, spontaneous $\mathrm{CP}$ violation (SCPV) can only occur if the radiative corrections are taken into account leading to a very light Higgs boson which has been discarded by the experiment ${ }^{24}$. In the NMSSM, spontaneous CP violation can occur even at the tree level, for those models without a discrete $Z_{3}$ symmetry ${ }^{25,26}$. For models which has $Z_{3}$ symmetry, the NMSSM can not produce SCPV at the tree level ${ }^{27}$. SCPV can only occur if the stop quark masses at the one-loop effective potential are non-degenerate ${ }^{28}$. However, at the tree level, unlike the MSSM, explicit CP violation is possible in the NMSSM 29,30 .

In the literature, the NMSSM has been studied through many researches including the effects of one-loop radiative corrections due to various particles, and their superpartners $31,32,33,34,35$, and the analysis of the Higgs potential with arbitrary number of Higgs singlets ${ }^{36}$. In the earlier studies, the corrections to mass matrices of the top and stop quarks have been calculated with either the one-loop effective potential or the renormalization group approach ${ }^{31}$. Among recent works the authors of Ref. [32] particularly addressed the effects of the explicit CP violation with the emphasis on the charged Higgs boson in the NMSSM, using the effective potential method, for which case the CP violating phases are induced from the stop and sbottom quark masses. This analysis is extended by including the contribution of the charginos ${ }^{33}$, and of the neutralinos ${ }^{34}$ to the one-loop effective potential, where the tree level $\mathrm{CP}$ violating phase is chosen to be equal to the one at the loop-level, and the complex phases of the chargino and neutralino contributions in the radiative corrections are taken into consideration.

The radiatively induced CP violation effects in the NMSSM has also been analyzed with renormalization group improvement ${ }^{37}$. It has been shown in Ref. [37] that the renormalization group analysis of radiative symmetry breaking always leads to $\tan \beta$ values that are larger than 1 . Indeed this result is a general feature of all phenomenologically viable theries that incorporate low energy supersymmetry and radiative breaking of the electroweak supersymmetry. More recent analysis on the Higgs mass spectrum motivated by the renormalization group analysis shows that to prevent the mass splitting between the light and heavy Higgs bosons from becoming too large, the value of $\tan \beta$ should be kept moderate $(\lesssim 10)^{38}$, which we shall also assume in this work.

In this work our aim is to investigate the CP violation capability of the NMSSM. Therefore, we limit our analysis to the effective potential with no renormalization group improvement. This accuracy has proven sufficient in obtaining the observable effects of explicit CP violation on the Higgs masses ${ }^{32}$. In doing this, we will keep the value of $\tan \beta$ moderate $(\lesssim 10)$, as favoured by the renormalization group analysis of [38], which is a well-motivated parameter regime for the model under concern (e.g, $\tan \beta=1.5$ is allowed within the framework of NMSSM ${ }^{37}$ ). For the purpose of showing the mass spectra, we shall take $\tan \beta=2$. To give feeling of the sensitivity 
of the mass on $\tan \beta$, we shall also consider $\tan \beta=10$ regime.

In the following we will study the radiatively corrected Higgs masses and mixings, taking into account of the $\mathrm{CP}$ violation effects, in the parameter space allowed by the electron electric dipole moment (eEDM) constraint. We will base our calculations to those of Ref. [32]. The main difference with the previous work ${ }^{32}$ springs from the fact that in our analysis the $\mathrm{CP}$ violating phases at the tree level and at the one-loop are not equal. Therefore, we analyze the mass spectra at the tree and one-loop levels, as well as the CP odd components of the Higgs boson by considering the effects of physical phases seperately. In the numerical analysis, we particularly focus on the real and complex cases of the tree-level coupling $\lambda$. Analyzing these specific cases for various parameter planes, we search for the impacts of the tree and one-loop level contributions on the Higgs sector which give the opportunity of comparing the results. In our analysis, we focus on the regions of the parameter space which are allowed by the eEDM constraint.

The organization of the paper is as follows: In Section 2, we study the Higgs masses and the one-loop eEDM in the NMSSM. In Section 3, we discuss two special cases of $\lambda$ for two different values of $v_{s}$, and $A_{t}$ at $\tan \beta=2$, and $\tan \beta=10$ regimes. In Section 4, we conclude the work.

\section{The Model}

The Higgs potential of the model at the tree level is given by :

$$
\begin{aligned}
V_{0} & =m_{H_{d}}^{2}\left|H_{d}\right|^{2}+m_{H_{u}}^{2}\left|H_{u}\right|^{2}+m_{S}^{2}|S|^{2}-\left[\lambda A_{\lambda} H_{d} H_{u} S+\frac{k}{3} A_{k} S^{3}+h . c\right] \\
& +|\lambda|^{2}\left[\left(\left|H_{d}\right|^{2}+\left|H_{u}\right|^{2}\right)|S|^{2}+\left|H_{d} H_{u}\right|^{2}\right]+|k|^{2}|S|^{4}-\left(\lambda k^{*} H_{d} H_{u} S^{* 2}+h . c\right) \\
& +\frac{1}{8}\left(g_{1}^{2}+g_{2}^{2}\right)\left(\left|H_{d}\right|^{2}-\left|H_{u}\right|^{2}\right)^{2}
\end{aligned}
$$

where the first, second and the third lines represent the soft SUSY breaking terms, $\mathrm{F}$ and $\mathrm{D}$ term contributions, respectively. Here, $A_{\lambda}$, and $A_{k}$ are the for the trilinear soft SUSY breaking terms.

Assuming $\lambda A_{\lambda}$, and $k A_{k}$ are real and positive, the phase between $\lambda$ and $k^{*}$ is given by:

$$
\varphi_{k \lambda}=\operatorname{Arg}\left[\lambda k^{*}\right]=\varphi_{\lambda}-\varphi_{k}
$$

and this phase forms the source of CP violation at the tree level in Eq. (1).

After electroweak breaking, the Higgs doublets and one Higgs singlet in (1) can be expanded as:

$$
\begin{aligned}
& H_{d}=\left(\begin{array}{c}
H_{d}^{0} \\
H_{d}^{-}
\end{array}\right)=\left(\begin{array}{c}
v_{d}+\phi_{1}+i \varphi_{1} \\
H_{d}^{-}
\end{array}\right) \\
& H_{u}=\left(\begin{array}{c}
H_{u}^{+} \\
H_{u}^{0}
\end{array}\right)=\left(\begin{array}{c}
H_{u}^{+} \\
v_{u}+\phi_{2}+i \varphi_{2}
\end{array}\right)
\end{aligned}
$$

and

$$
S=v_{s}+\phi_{s}+i \varphi_{s}
$$

As usual, we calculate the Higgs masses and their mixings up to one loop accuracy via

$$
M^{2}=\left(\frac{\partial^{2} V}{\partial \chi_{i} \partial \chi_{j}}\right)_{0}, \text { where } \chi_{i} \in \mathcal{B}=\left\{\phi_{1}, \phi_{2}, \varphi_{1}, \varphi_{2}, \phi_{s}, \varphi_{s}\right\}
$$


Here, $V \equiv V_{0}+V_{1-\text { loop }}$ is the radiatively corrected Higgs potential ${ }^{33}$, and as we mentioned before we take into account the top-stop and bottom-sbottom loop corrections.

The stop and sbottom mass-squared eigenvalues are given by:

$$
\begin{aligned}
& m_{\tilde{t}_{1,2}}^{2}=m_{t}^{2}+\frac{1}{2}\left(M_{Q}^{2}+M_{T}^{2}\right) \pm \Delta_{\tilde{t}}^{2}, \\
& m_{\tilde{b}_{1,2}}^{2}=m_{b}^{2}+\frac{1}{2}\left(M_{Q}^{2}+M_{T}^{2}\right) \pm \Delta_{\tilde{b}}^{2},
\end{aligned}
$$

where the stop and sbottom mass-splittings read as:

$$
\begin{aligned}
\Delta_{\tilde{t}}^{2} & =\sqrt{\frac{1}{4}\left(M_{Q}^{2}-M_{T}^{2}\right)^{2}+m_{t}^{2}\left(A_{t}^{2}+\lambda^{2} v_{s}^{2}\left(t_{\beta}^{-1}\right)^{2}+2 A_{t} \lambda v_{s} t_{\beta}^{-1} \cos \varphi_{\lambda t}\right)}, \\
\Delta_{\tilde{b}}^{2} & =\sqrt{\frac{1}{4}\left(M_{Q}^{2}-M_{T}^{2}\right)^{2}+m_{b}^{2}\left(A_{b}^{2}+\lambda^{2} v_{s}^{2}\left(t_{\beta}\right)^{2}+2 A_{b} \lambda v_{s} t_{\beta} \cos \varphi_{\lambda t}\right)} .
\end{aligned}
$$

Here, $t_{\beta}=\tan \beta, t_{\beta}^{-1}=\cot \beta$. For convenience, we set the soft SUSY breaking scalarquark masses as $M_{\tilde{Q}}=M_{\tilde{u}}=M_{\tilde{d}}$, and the squark trilinear couplings as $A_{t}=A_{b}$.

The stop and sbottom mass splittings depend explicitely on the total CP violation angle $\varphi_{\lambda t}$ between $A_{t}=A_{b}$, and $\lambda$

$$
\varphi_{\lambda t}=\operatorname{Arg}\left[\lambda A_{t}\right]
$$

which forms the source of CP violation at the tree and one-loop level Higgs potential of the NMSSM.

The $(5 \times 5)$ dimensional Higgs mass-squared matrix can be expressed as:

$$
M_{i j}=M_{i j}+\Delta M_{i j} .
$$

Here, $M_{i j}$ comes from the tree-level potential, whereas $\Delta M_{i j}$ from the stop and sbottom contributions at the one-loop level ${ }^{32}$.

The elements of the mass matrix at the tree level are are given by:

$$
\begin{aligned}
& M_{11}=\left[m_{Z} \cos \beta\right]^{2}+\left[m_{A} \sin \beta\right]^{2}, \\
& M_{12}=-\left[m_{Z}^{2}+m_{A}^{2}-2 \lambda^{2} v^{2}\right] \sin \beta \cos \beta \\
& M_{13}=0, \\
& M_{14}=-\left(\frac{v}{v_{s}}\right)\left[m_{A}^{2} \sin ^{2} \beta \cos \beta-2\left(\lambda^{2} v_{s}^{2}\right) \cos \beta+\left(\frac{k}{\lambda}\right)\left(\lambda^{2} v_{s}^{2}\right) \cos \varphi_{\lambda t} \sin \beta\right], \\
& M_{15}=-3\left(\frac{v}{v_{s}}\right)\left[\left(\frac{k}{\lambda}\right)\left(\lambda^{2} v_{s}^{2}\right) \sin \beta \sin \varphi_{\lambda t}\right], \\
& M_{22}=\left[m_{Z} \sin \beta\right]^{2}+\left[m_{A} \cos \beta\right]^{2}, \\
& M_{23}=0, \\
& M_{24}=-\left(\frac{v}{v_{s}}\right)\left[m_{A}^{2} \sin \beta \cos ^{2} \beta-2\left(\lambda^{2} v_{s}^{2}\right) \sin \beta+\left(\frac{k}{\lambda}\right)\left(\lambda^{2} v_{s}^{2}\right) \cos \varphi_{k \lambda} \cos \beta\right], \\
& M_{25}=M_{52}=-3\left(\frac{v}{v_{s}}\right)\left[\left(\frac{k}{\lambda}\right)\left(\lambda^{2} v_{s}^{2}\right) \sin \varphi_{\lambda t} \cos \beta\right],
\end{aligned}
$$




$$
\begin{aligned}
M_{33} & =m_{A}^{2} \\
M_{34} & =\left(\frac{v}{v_{s}}\right)\left[\left(\frac{k}{\lambda}\right)\left(\lambda^{2} v_{s}^{2}\right) \sin \varphi_{\lambda t}\right] \\
M_{35} & =\left(\frac{v}{v_{s}}\right)\left[m_{A}^{2} \sin \beta \cos \beta-3\left(\frac{k}{\lambda}\right)\left(\lambda^{2} v_{s}^{2}\right) \cos \varphi_{\lambda t}\right] \\
M_{44} & =\left(\frac{v^{2}}{v_{s}^{2}}\right) \sin \beta \cos \beta\left(\sin \beta \cos \beta m_{A}^{2}-\left(\frac{k}{\lambda}\right)\left(\lambda^{2} v_{s}^{2}\right) \cos \varphi_{\lambda t}\right] \\
& +\left(\frac{k^{2}}{\lambda^{2}}\right)\left(\lambda^{2} v_{s}^{2}\right)-\left(\frac{k}{\lambda}\right)\left(\lambda v_{s}\right) A_{k}, \\
M_{45} & =2 \frac{k}{\lambda}\left[\left(\lambda^{2} v_{s}^{2}\right) \sin 2 \beta \sin \varphi_{\lambda t}\right] \\
M_{55} & =\left(\frac{v^{2}}{v_{s}^{2}}\right) \sin \beta \cos \beta\left[m_{A}^{2} \sin \beta \cos \beta+3\left(\frac{k}{\lambda}\right)\left(\lambda^{2} v_{s}^{2}\right) \cos \varphi_{\lambda t}\right] \\
& +3\left(\frac{k}{\lambda}\right)\left(\lambda v_{s}\right) A_{k},
\end{aligned}
$$

where

$$
m_{A}^{2}=\frac{\left(\lambda v_{s}\right)\left[A_{\lambda}+k v_{s} \cos \varphi_{\lambda t}\right]}{\sin \beta \cos \beta} .
$$

The radiative correction terms due to the stop and sbottom corrections at the one-loop $\left(\Delta M_{i j}\right)$ can be found in the work of Ref. [32]. However, as mentioned before, we differ from Ref. [32] in the sense that, in our analysis, the CP violating phase at the tree-level is not equal to the one at the one-loop.

In our analysis, we will particularly concentrate on the lightest Higgs boson, whose mass can be obtained by the diagonalization of the Higgs mass-squared matrix by the similarity transformation:

$$
\mathcal{R} M_{H}^{2} \mathcal{R}^{T}=\operatorname{diag}\left(m_{h_{1}}^{2}, m_{h_{2}}^{2}, m_{h_{3}}^{2}, m_{h_{4}}^{2}, m_{h_{5}}^{2}\right),
$$

where $\mathcal{R R}^{T}=1$. Here, we define $h_{5}$ to be the lightest of all five Higgs bosons .

The mass eigenstates of the lightest Higgs boson $\left(h_{5}\right)$ can then be decomposed in terms of the basis elements as:

$$
h_{5}=\sum_{i=1}^{5} \mathcal{R}_{5 i} \Phi_{i},
$$

where $\Phi_{1}, \Phi_{2}, \Phi_{3}, \Phi_{4}, \Phi_{5}$ correspond respectively, $\phi_{1}, \phi_{2}, \sin \beta \varphi_{1}+\cos \beta \varphi_{2}, \phi_{s}$ and $\varphi_{s}$ components of the Higgs boson under consideration.

From Eq. (13), we define the dimensionless quantity $\rho_{i}$,

$$
\rho_{i}=100 \times\left|\mathcal{R}_{5 i}\right|^{2} i=1,2,3,4,5,
$$

which is a measure of the percentage $\mathrm{CP}$ component of a given mass-eigenstate Higgs boson. Therefore, in (14) for instance, $\rho_{3}$ and $\rho_{5}$ are measures of the percentage $\mathrm{CP}$ odd components of $h_{5}$. 
The main contributions to the one-loop eEDM come from the neutralino, and chargino exchanges which can be expressed as:

$$
\left(\frac{d_{e}}{e}\right)^{1-\text { loop }}=\left(\frac{d_{e}}{e}\right)^{\tilde{e}-\chi_{i}^{0}}+\left(\frac{d_{e}}{e}\right)^{\tilde{\nu_{e}}-\chi_{i}^{+}}
$$

By taking into account of the neutralino-selectron interaction, the neutralino contribution to the eEDM can be written as:

$$
\left(\frac{d_{e}}{e}\right)^{\tilde{e}-\chi_{i}^{0}}=\frac{\alpha}{4 \pi s_{W}^{2}}\left\{\sum_{k=1}^{2} \sum_{i=1}^{5} \operatorname{Im}\left[\eta_{e_{i k}}\right] M_{\chi_{i}^{0}, \tilde{e}_{k}} \mathcal{B}\left(M_{\chi_{i}^{0}, \tilde{e}_{k}}^{2}\right)\right\}
$$

which is very similar to that of MSSM ${ }^{39}$, except for the fact that the neutralino sector now extends to $5 \times 5$ mass matrix in the NMSSM, due to the presence of the additional gauge singlet. By convention, in Eq. (16) and in the formalism below, we use the short-hand notation, for the generic indices $\alpha$ and $\rho, M_{\alpha, \rho} \equiv \frac{m_{\alpha}}{m_{\rho}}$, and $s_{W}\left(c_{W}\right)=\sin \theta_{W}\left(\cos \theta_{W}\right), \quad c_{\beta}=\cos \beta, \quad t_{W}=\tan \theta_{W}$. sion:

In Eq. (16) $\eta_{e_{i k}}$ is the neutralino vertex which is given by the following expres-

$$
\begin{aligned}
\eta_{e_{i k}} & =-\left[\left(t_{W} \mathcal{N}_{1 i}+\mathcal{N}_{2 i}\right) \tilde{\mathcal{S}}_{e 1 k}^{*}+\frac{M_{e, w}}{c_{\beta}} \mathcal{N}_{3 i} \tilde{\mathcal{S}}_{e 2 k}^{*}\right] \\
& \times\left[t_{W} \mathcal{N}_{1 i} \tilde{\mathcal{S}}_{e 2 k}+\frac{M_{e, w}}{2 c_{\beta}} \mathcal{N}_{3 i} \tilde{\mathcal{S}}_{e 1 k}\right]
\end{aligned}
$$

where $\mathcal{N}$ is the unitary matrix diagonalizing the neutralino matrix: $\mathcal{N}^{T} M_{\chi^{0}} \mathcal{N}=$ $\operatorname{diag}\left(m_{\chi_{1}^{0}}, \cdots, m_{\chi_{5}^{0}}\right)$. The eigenstates $\left(\tilde{e}_{1}, \tilde{e}_{2}\right)$ in Eq. (17) can be obtained via the unitary rotation: $\tilde{\mathcal{S}}_{e}^{\dagger} \widetilde{M}_{e}^{2} \tilde{\mathcal{S}}_{e}=\operatorname{diag}\left(m_{\tilde{e}_{1}}^{2}, m_{\tilde{e}_{2}}^{2}\right)$.

On the other hand, taking into account of the sneutrino-chargino interaction, the chargino contribution to eEDM reads as ${ }^{39}$ :

$$
\left(\frac{d_{e}}{e}\right)^{\tilde{\nu_{e}}-\chi_{i}^{+}}=\frac{\alpha}{4 \pi s_{W}^{2}}\left\{\frac{M_{e, w}}{\sqrt{2} c_{\beta} m_{\tilde{\nu}_{e}}^{2}} \sum_{i=1}^{2} m_{\chi_{i}^{+}} \operatorname{Im}\left[\mathcal{U}_{i 2}^{*} \mathcal{V}_{i 1}^{*}\right] \mathcal{A}\left(M_{\chi_{i}^{+}, \tilde{\nu}_{e}}^{2}\right)\right\}
$$

where $\mathcal{U}$ and $\mathcal{V}$ are the unitary matrices diagonalizing the chargino mass matrix: $\mathcal{U}^{*} M_{C} \mathcal{V}^{-1}=\operatorname{diag}\left(m_{\chi_{1}^{+}}, m_{\chi_{2}^{+}}\right)$. In Eqns. (16)-(18), $\mathcal{B}$ and $\mathcal{A}$ are the the loop functions ${ }^{39}$.

We would like to note that we take into account one loop contributions to eEDM. It was pointed out in Ref. [40] that in certain regions of MSSM parameter space certain two-loop contributions can not be non-negligible. However, these two-loop contributions become sizeable only at high $\tan \beta(\tan \beta \gtrsim 30)$. In this work we have restricted our attention to moderate $\tan \beta$ values $(\tan \beta \lesssim 10$ ) which is a well-motivated parameter regime for the model under consideration ${ }^{37}$. Hence the two-loop eEDM will not provide significant contribution in our analysis.

Eq. (15), possesses the sources of CP violation through

(i) the gaugino masses

$$
\left(m_{2}, m_{1}\right) \rightarrow\left(m_{2} e^{i \varphi_{2}}, m_{1} e^{i \varphi_{1}}\right)
$$


which involves gaugino $\left(\varphi_{1}\right)$ and the $\mathrm{SU}(2)$ gaugino $\left(\varphi_{2}\right)$ phases in neutralino and chargino mass matrices.

(ii) the complex selectron trilinear coupling

$$
\left(A_{e}\right) \rightarrow\left(A_{e} e^{i \varphi_{e}}\right),
$$

Therefore, the phases of the stop and sbottom trilinear couplings $\left(\varphi_{A_{t}}=\varphi_{A_{b}}\right)$, the phases of the gaugino masses $\left(\varphi_{1}\right.$, and $\left.\varphi_{2}\right)$, the phases of the the Higgs potential, at tree and at the one-loop level $\left(\varphi_{k \lambda}\right.$, and $\varphi_{\lambda t}$, respectively) form the CP violating sources in the full parameter space. In the following, we will perform a numerical study to determine the eEDM, the effects of the physical phases of the model on the mass and on the CP-odd components $\left(\rho_{3}\right.$ and $\left.\rho_{5}\right)$ of $h_{5}$, in the parameter space allowed by the eEDM constraint.

\section{Numerical Analysis}

In this section we will consider various parameter planes to adress the issue of whether or not the various parameters would lead to a large amount of $\mathrm{CP}$ violation opportunities. As seen in the previous section, there are two physical phases which contribute to the Higgs boson mass matrix. Besides these physical phases, the free parameters appearing at the tree and one-loop levels are $\tan \beta, A_{k}, A_{\lambda}, k, A_{k}, v_{s}$. $A_{t}$ and $A_{b}$.

In our analysis, we particularly concentrate on $h_{5}$, and analyze various parameter planes, in the parameter space allowed by the eEDM constraint. In doing this, we use the present experimental upper bound of the eEDM ${ }^{41,42}$ :

$$
d_{e}<4.3 \times 10^{-27} \text { e.cm },
$$

A convinient way to observe the effects of the eEDM constraint, is via the dimensionless quantity:

$$
\mathrm{eEDM}=\frac{\left[d_{e} / e\right]^{t h}}{\left[d_{e} / e\right]^{e x p}},
$$

which measures the fractional enhancement or suppression of the eEDM with respect to its experimental value.

In the numerical analysis, for the purpose of definiteness, we set: $(i)$ the trilinear couplings $A_{t}=A_{b}(i i) A_{\lambda}=v_{s}$, and $A_{k}=100 \mathrm{GeV}$. We focus on the values of $k$, and $\lambda$ that are favored by the renormalization group equations in the NMSSM ${ }^{37}$. In doing this, we fix the $k$ parameter to be near its fixed point value (i.e. we choose $k=0.63$ ), and we focus on the allowed values of $\lambda$, in the $0.12 \lesssim \lambda \lesssim 0.82$ interval.

We first search whether or not the eEDM is consistent with the present experimental bounds in the above mentioned intervals provided that the gaugino masses are of $\mathcal{O}(\mathrm{TeV})$. Namely, we take: $(i)$ the gaugino masses as $M_{2}=2000 \mathrm{GeV}$, $M_{1}=1000 \mathrm{GeV},(i i)$ the slepton masses from the neutralino and chargino sector as $M_{\tilde{L}}=1500 \mathrm{GeV}$, and $M_{\tilde{R}}=1000 \mathrm{GeV}$.

As mentioned in the Introduction, we take into account of not very large values of $\tan \beta$ (i.e. $\tan \beta \lesssim 10$ ). For the purpose of showing the mass spectra, we shall take $\tan \beta=2$. To give feeling of the sensitivity of the mass on $\tan \beta$, we shall also consider $\tan \beta=10$ regime. In the numerical analysis, we concentrate on two specific cases: in the first part, we carry out the analysis by letting $\lambda$ of a real parameter, then in the second part we take into account of the case for which $\lambda$ is complex. 


\subsection{The Case of Real $\lambda$}

In the first part of our analysis, we take $\lambda$ as a real parameter $\left(\varphi_{\lambda}=0\right)$, and let all the other phases in the theory of being complex. Namely, $\varphi_{A_{t}}=\varphi_{A_{b}}=\varphi_{A_{e}}=\varphi_{1}=$ $\varphi_{2}=\varphi$. In Fig. 1, we show the dependence of $|e E D M|$ on $\varphi$ and $\lambda$ for selected values of the vacuum expectation value of the singlet $\left(v_{s}\right)$ when $\tan \beta=2$ (left panel) and $\tan \beta=10$ (right panel). In the figure, we obtain 3-dimensional surfaces for each value of $v_{s}$, which we choose within the $v \lesssim v_{s} \lesssim 4 v$ interval. For instance, the top surface corresponds to $v_{s}=4 v$, whereas the bottom represents $v_{s}=v$. Here, $\varphi$ changes from 0 to $2 \pi$, and $\lambda$ from 0.12 to 0.82 . As both panels of Fig. 1 suggest the upper bound on $|e E D M|$ gradually increases, with the increasing values of $\lambda$, and of $v_{s}$. One notes that the present bound of $|e E D M|$ is consistent with the present experimental bound in the full $\varphi$ range, for all values $v \lesssim v_{s} \lesssim 4 v$, and $0.12 \lesssim \lambda \lesssim 0.82$, at both $\tan \beta=2$ (left panel) and $\tan \beta=10$ (right panel) regimes, provided that the gaugino masses are of $\mathcal{O}(\mathrm{TeV})$.
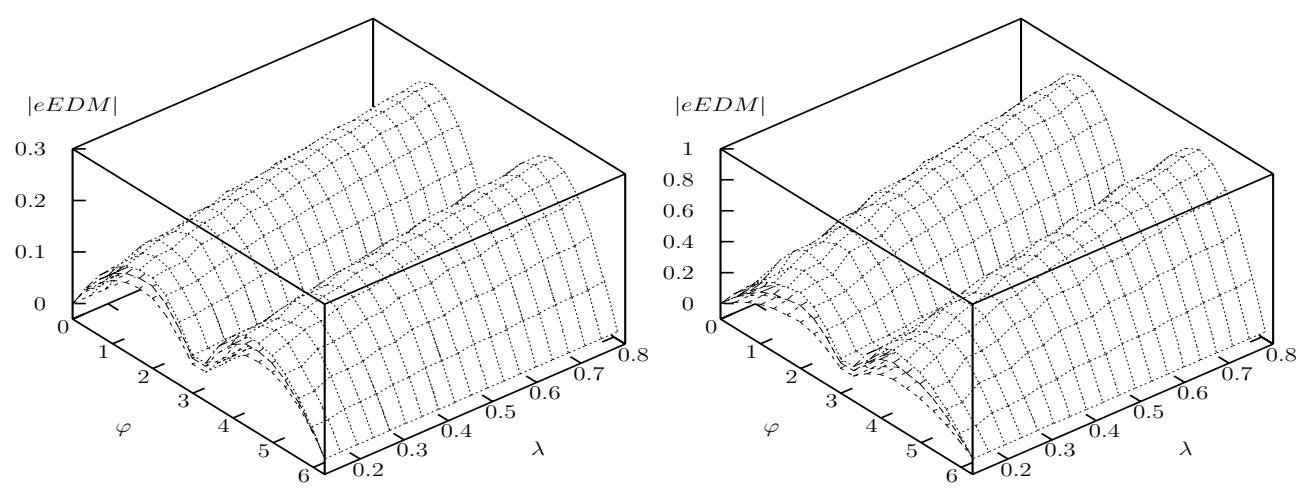

Fig. 1. The dependence of $|e E D M|$ on $\varphi$ and $\lambda$ for selected values of the vacuum expectation value of the singlet when $\tan \beta=2$ (left panel) and $\tan \beta=10$ (right panel).

The analysis of Fig. 1 gives a general idea of the parameter domain of the $|e E D M|$ and $\varphi-\lambda$ plane, when $\varphi_{\lambda}=0$, and $\varphi$ changes in its full range. With this input in mind, we choose two values of $\lambda(\lambda=0.12$, and $\lambda=0.45)$, and of $v_{s}$ $\left(v_{s}=v\right.$ and $\left.v_{s}=3 v\right)$, in the parameter space allowed by the eEDM constraint, corresponding to the low and high values of $\lambda$ and $v_{s}$, respectively, to analyze the dependence of the mass $\left(m_{h_{5}}\right)$ at the tree and one-loop levels, and of the CP odd components of the lightest Higgs boson $\left(h_{5}\right)$ on $\varphi$ at both $\tan \beta$ regimes.

We would like to note that, in the remaining part of the analysis the variation of $m_{h_{5}}$ as well as $\rho_{3}$ and $\rho_{5}$, with $\varphi$ is displayed for several values of $A_{t}$ taking into account of the strong dependence of the radiative corrections on the stop splitting, as will be seen in Eq. (22).

Therefore, in each of the following plots, we first consider the case for which $A_{t}$ and $v_{s}$ are of comparable size, (i.e, $A_{t}=v_{s}$ ) when $v_{s}=175 \mathrm{GeV}$, and $v_{s}=525 \mathrm{GeV}$. Next, to determine how the increase in $A_{t}$ affects the radiative corrections, we concentrate on two specific values of $A_{t}$ corresponding to $A_{t}=1050 \mathrm{GeV}$, and $A_{t}=1400 \mathrm{GeV}$ for both $v_{s}=175 \mathrm{GeV}$ and $v_{s}=525 \mathrm{GeV}$ cases.

In Fig. 2, we show the dependence of $m_{h_{5}}$ (at tree and at one-loop levels) on $\varphi$ at $\tan \beta=2$ (left panel) and $\tan \beta=10$ (right panel), when $v_{s}=v=175 \mathrm{GeV}$, and $\lambda=0.12$, for selected values of $A_{t}$. In both panels of Fig. 2, the three upper curves with respect to the mid-point, from bottom to top, represent $A_{t}=v_{s}=175 \mathrm{GeV}$, 
$A_{t}=1050 \mathrm{GeV}$, and $A_{t}=1400 \mathrm{GeV}$ values of $A_{t}$ respectively, whereas the lowest curve is for the tree-level.
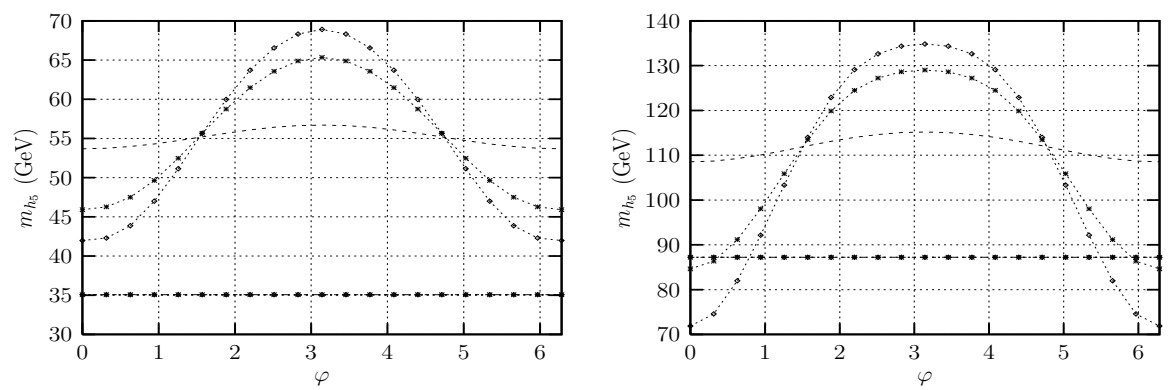

Fig. 2. The dependence of $m_{h_{5}}$ on $\varphi$, for selected values of $A_{t}$, at $\tan \beta=2$ (left panel), and $\tan \beta=10$ (right panel) when $v_{s}=175 \mathrm{GeV}$ and $\lambda=0.12$. Here, the three upper curves with respect to the mid-point, from bottom to top, are for $A_{t}=v_{s}=175 \mathrm{GeV}, A_{t}=1050 \mathrm{GeV}$, and $A_{t}=1400 \mathrm{GeV}$, respectively, whereas the lowest curve is for the tree-level.

As Fig. 2 suggests when $\lambda=0.12$, and $v_{s}=175 \mathrm{GeV}, m_{h_{5}}$ grows from $(42,46,54)$ to $(69,65,56) \mathrm{GeV}$ at $\tan \beta=2$, and from $(71,85,109)$ to $(135,130,115)$ $\mathrm{GeV}$ at $\tan \beta=10$, for the values of $A_{t}=1400 \mathrm{GeV}$ (the top curve with respect to the mid-point), $A_{t}=1050 \mathrm{GeV}$ (the second curve below the top curve), and $A_{t}=v_{s}$ (the third curve below the top curve), respectively, as $\varphi$ ranges from 0 to $\pi$. On the other hand, remaining around $35 \mathrm{GeV}$ for $\tan \beta=2, m_{h_{5}}$ grows up until $87 \mathrm{GeV}$, at the tree level, for $\tan \beta=10$. It can also be observed from Fig. 2 that $m_{h_{5}}$ is quite sensitive to the variations in $\varphi$ for $\lambda=0.12$, and $v_{s}=175 \mathrm{GeV}$ for both $\tan \beta=2$ (left panel) and $\tan \beta=10$ (right panel) regimes. One notes that the splittings between the tree and one-loop spectra are quite small. However, as $v_{s}$ gets higher values, the strength of the radiative corrections are affected, which causes larger splittings between the tree and one-loop mass spectra.
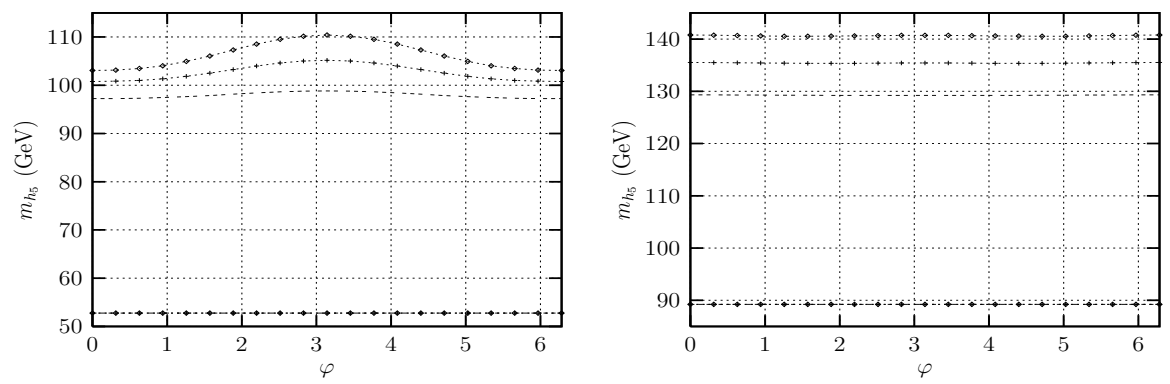

Fig. 3. The dependence of $m_{h_{5}}$ on $\varphi$, for selected values of $A_{t}$, at $\tan \beta=2$ (left panel), and $\tan \beta=10$ (right panel), when $v_{s}=525 \mathrm{GeV}$ and $\lambda=0.12$. Here, the bottom, the middle and the top curves, are for $A_{t}=v_{s}=525 \mathrm{GeV}, A_{t}=1050 \mathrm{GeV}$, and $A_{t}=1400 \mathrm{GeV}$ values, whereas the lowest curve is for the tree level.

For instance, in Fig. 3, we show the dependence of $m_{h_{5}}$ on $\varphi$, for $v_{s}=3 v=$ $525 \mathrm{GeV}$, and $\lambda=0.12$. In Fig. 3 , we select three specific values of $A_{t}$ : namely $A_{t}=1400 \mathrm{GeV}$ (the top curve), $A_{t}=1050 \mathrm{GeV}$ (the second curve below the top curve), $A_{t}=v_{s} \mathrm{GeV}$ (the third curve below the top curve) Here, the lowest curve 
represents $m_{h_{5}}$ at the tree-level. It can be seen from the left panel of Fig. 3 that as $v_{s}$ increases the gap between the tree and the one-loop levels enlarges. For instance, being around at most $\sim 20 \mathrm{GeV}$ for $v_{s}=v$ and $A_{t}=v_{s}$ (left panel of Fig. 2), it rises to $\sim 50 \mathrm{GeV}$ for $v_{s}=3 v$ at $\tan \beta=2$ (left panel of Fig. 3). This behaviour occurs also in the $\tan \beta=10$ regime for which case the gap between the tree and one-loop levels increases (right panel of Fig. 3).

A comparative analysis of left panels of Fig. 2 and Fig. 3 shows that when $A_{t}$ and $v_{s}$ are of comparible size, the variation of $m_{h_{5}}$ with $\varphi$ is much more slower as compared to the cases for which $A_{t}>v_{s}\left(A_{t}=1050 \mathrm{GeV}\right.$, and $\left.A_{t}=1400 \mathrm{GeV}\right)$. This saturation effect can be understood by observing that the radiative corrections depend strongly on the stop splitting $\Delta_{\tilde{t}}^{2}$, which depends explicitely on $\varphi_{\lambda t}$ such that

$$
\delta=\Delta_{\tilde{t}}^{2}(\pi) / \Delta_{\tilde{t}}^{2}(0)=\frac{\left|A_{t}\right|-|\lambda| v_{s} \cot \beta}{\left|A_{t}\right|+|\lambda| v_{s} \cot \beta}=\frac{1-\frac{|\lambda| \cot \beta}{\left|A_{t}\right| / v_{s}}}{1+\frac{|\lambda| \cot \beta}{\left|A_{t}\right| / v_{s}}}
$$

This quantity particularly implies that the strength of the radiative corrections modify from one $\mathrm{CP}$ conserving point to the next. It can also be seen from (23) that when $\left|A_{t}\right|$ and $v_{s}$, are of comparible size, as $\tan \beta$ increases, $|\lambda| \cot \beta$ decreases and indeed, $\delta$ approaches to unity in the high $\tan \beta$ limit. One notes that the tree level mass of the model is proportional to $\lambda^{2} v_{s}^{2}$, whereas the CP violating entries of the radiative corrections of the Higgs mass-squared matrix grow with the term $\left|A_{t}\right| v_{s} \lambda$. Naturally, with the increase in $A_{t},\left|A_{t}\right| v_{s} \lambda$, term also increases. That is the radiative corrections enhance through the $A_{t}$ term, which does not cause too big splittings among the radiative corrections, when $v_{s}=v$ and $\lambda=0.12$. However, as $v_{s}$ gets higher values, the seperation from the tree-level enlarges.

In Fig. 4, we show the dependence of CP-odd components $\left(\rho_{3}\right.$ and $\left.\rho_{5}\right)$ of $h_{5}$ on $\varphi$, for $\lambda=0.12$, and $v_{s}=v=175 \mathrm{GeV}$, when $\tan \beta=2$ (left panel) and $\tan \beta=10$ (right panel). In both panels the upper and the lower curves represent the $\rho_{3}$, and $\rho_{5}$ components of $h_{5}$, respectively. We select three values of $A_{t}: A_{t}=$ $v_{s}=175 \mathrm{GeV}, A_{t}=1050 \mathrm{GeV}, A_{t}=1400 \mathrm{GeV}$, from top to bottom for the upper curves $\left(\rho_{3}\right)$, and from bottom to top for the lower curves $\left(\rho_{5}\right)$.
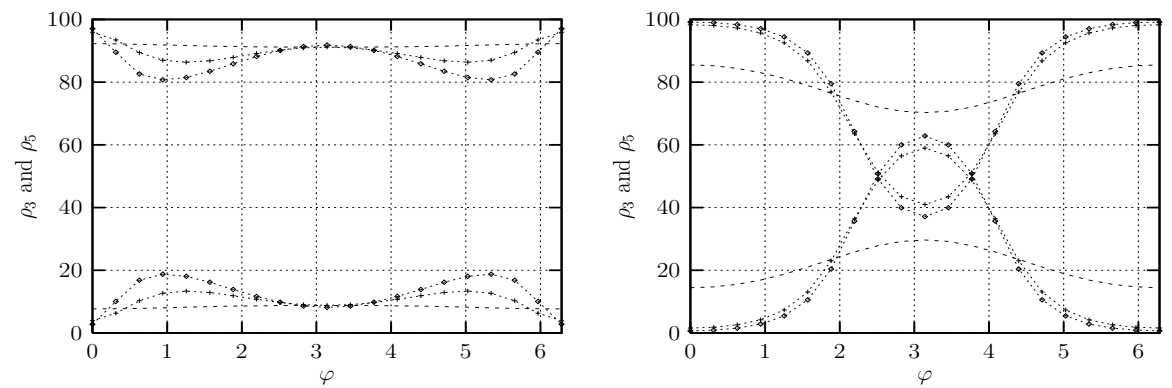

Fig. 4. The dependence of $\rho_{3}$ (the upper curves) and $\rho_{5}$ (the lower curves) on $\varphi$ for $v_{s}=175 \mathrm{GeV}$, and $\lambda=0.12$, for selected values of $A_{t}$, when $\tan \beta=2$ (left panel), and $\tan \beta=10$ (right panel). Here, $A_{t}=v_{s}=175 \mathrm{GeV}, A_{t}=1050 \mathrm{GeV}, A_{t}=1400 \mathrm{GeV}$, from top to bottom for $\rho_{3}$, and from bottom to top for $\rho_{5}$.

As the left panel of Fig. 4 suggests, when $A_{t}=1400 \mathrm{GeV}$, and $A_{t}=1050 \mathrm{GeV}$ $h_{5}$ has $\% 98$ and $\% 96 \rho_{3}$ components, respectively, at $\tan \beta=2$ and $\varphi=0$. When $A_{t}=v_{s}, \rho_{3}$ decreases to $\% 92$. The left panel of the Fig. 4 also indicates that $\rho_{5}$ ranges in between $\% 2$ and $\% 20$, depending on the strength of $A_{t}$. On the other 
hand, as we observe from the right panel, when $\tan \beta=10, \rho_{3}$ rises nearly to the $\% 100$ line for $A_{t}=1050 \mathrm{GeV}$ (and for $A_{t}=1400 \mathrm{GeV}$, as well), and correspondingly $\rho_{5}$ remains in the vicinity of the $\% 0$ line at $\varphi=0$. For higher values of $v_{s}$, the $\rho_{5}$ component of $h_{5}$ increases, and this increase in the $\rho_{5}$ is compensated by $\rho_{3}$, as expected (see Fig. 5).
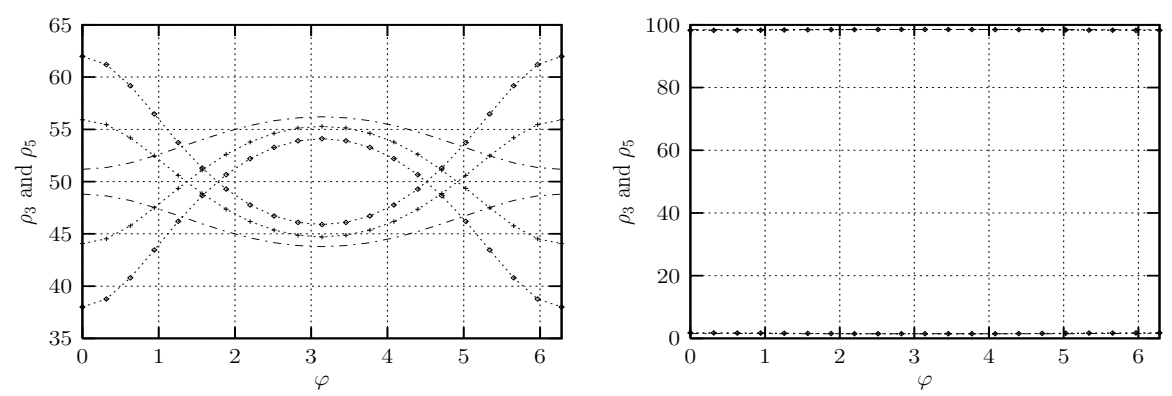

Fig. 5. The dependence of $\rho_{3}$ (the lower curves) and $\rho_{5}$ (the upper curves) on $\varphi$ for $v_{s}=525 \mathrm{GeV}$, and $\lambda=0.12$, for selected values of $A_{t}$, when $\tan \beta=2$ (left panel) and $\tan \beta=10$ (right panel). Here, $A_{t}=v_{s}=525 \mathrm{GeV}, A_{t}=1050 \mathrm{GeV}$ and $A_{t}=1400 \mathrm{GeV}$, from bottom to top for $\rho_{3}$, and from top to bottom for $\rho_{5}$, with respect to the mid-point.

In Fig. 5, we show the dependence of $\rho_{3}$ and $\rho_{5}$ components of $h_{5}$ on $\varphi$, for $\lambda=0.12$, and $v_{s}=3 v=525 \mathrm{GeV}$, when $\tan \beta=2$ (left panel), and $\tan \beta=10$ (right panel). Here, the lower and the upper curves represent the $\rho_{3}$, and $\rho_{5}$ components of $h_{5}$, respectively. Similar to the Fig. 4, in Fig. 5, we select three values of $A_{t}$ : $A_{t}=v_{s}, A_{t}=1050 \mathrm{GeV}, A_{t}=1400 \mathrm{GeV}$, from bottom to top for the lower curves $\left(\rho_{3}\right)$, and from top to bottom for the upper curves $\left(\rho_{5}\right)$, with respect to the midpoint. It can be noted from the left panel of Fig. 5 that as $v_{s}$ gets higher values the $\rho_{5}$ component of $h_{5}$ increases, with the decrease of its $\rho_{3}$ component, as compared to the previous case (see Fig. 4). For instance, when $\tan \beta=2$ (left panel of Fig. $5)$, and $A_{t}=1400 \mathrm{GeV}, \rho_{3}$ decreases to $\sim \% 63$ whereas $\rho_{5}$ increases to $\sim \% 37$ at $\varphi=0$. A more spectacular side of Fig. 5 arises for $\tan \beta=10$ (the right panel) where one observes a very slow variation of $\varphi$ as compared to the previous case (the right panel of Fig. 4).

A comparative analysis of Figs. 4 and 5 suggest that for small $\lambda(\lambda=0.12)$ and higher $v_{s}\left(v_{s}=3 v\right)$, the $\rho_{5}$ component of $h_{5}$ increases, and this increase is compensated by the decrease in $\rho_{3}$. Such kind of effect can be dominantly seen in the high $\tan \beta$ regime (see the right panel of Fig. 5 ).

One notes that in the MSSM there is a single CP-odd component, and naturally the results of the $\mathrm{CP}$-invariant theory are expected to be recovered at the $\mathrm{CP}$ conserving points $(\varphi=0, \pi, 2 \pi)^{43}$. However, there are two CP odd components in the NMSSM $\left(\rho_{3}\right.$ and $\left.\rho_{5}\right)$, and these two odd components mix each other at the $\mathrm{CP}$ conserving points, depending on the relative strengths of the other one-loop corrections. Therefore, one necessarily does not recover the results of the CPinvariant theory at the $\mathrm{CP}$ conserving points, due to the $\mathrm{CP}$ violating mixings of $\rho_{3}$ and $\rho_{5}$.

Until now, we have carried out our analysis for small values of $\lambda$, when $\varphi_{\lambda}=0$, and all the other phases in the theory are assumed to be complex. In the following, we take into account of higher values of $\lambda$. For this reason, we fix $\lambda=0.45$ and carry out the similar analysis for the second part of the work.

In Fig. 6, we show the dependence of $m_{h_{5}}$ (at tree and at one-loop levels) on $\varphi$ for $v_{s}=v=175 \mathrm{GeV}$, and $\lambda=0.45$, when $\tan \beta=2$ (left panel) and $\tan \beta=10$ (right panel). We again select three values of $A_{t}$, namely $A_{t}=1400 \mathrm{GeV}$ (the top curve 

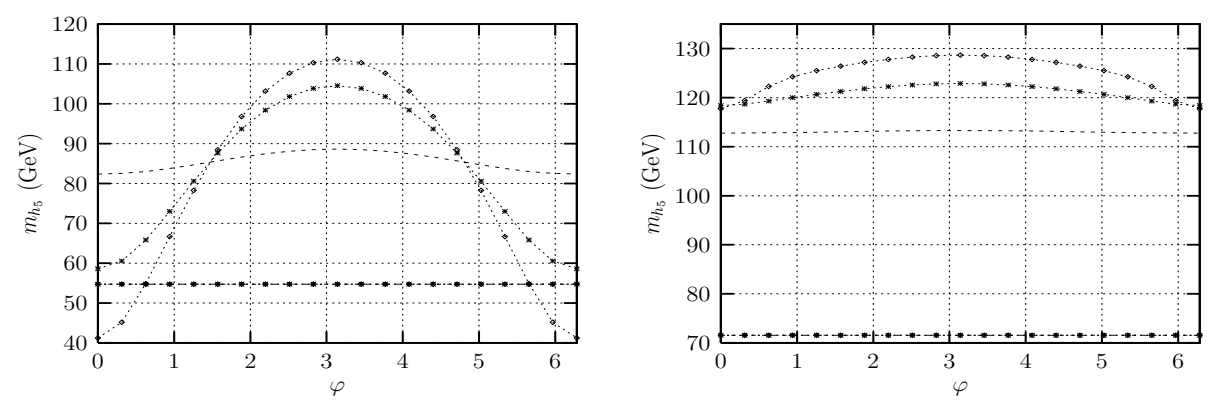

Fig. 6. The dependence of $m_{h_{5}}$ on $\varphi$, for selected values of $A_{t}$, at $\tan \beta=2$ (left panel), and $\tan \beta=10$ (right panel) when $v_{s}=\mathrm{v}$ and $\lambda=0.45$. Here, the three upper curves with respect to the mid-point, from bottom to top, are for $A_{t}=v_{s}=175 \mathrm{GeV}, A_{t}=1050 \mathrm{GeV}$, and $A_{t}=1400 \mathrm{GeV}$, respectively, whereas the lowest curve is for the tree level.

with respect to the mid-point), $A_{t}=1050 \mathrm{GeV}$ (the second curve below the top curve), $A_{t}=v_{s}=175 \mathrm{GeV}$ (the third curve below the top curve). Here, the lowest curve is for the tree-level. It can be observed from Fig. 6 that $m_{h_{5}}$ still remains sensitive to the variations in $\varphi$ for higher values of $\lambda(\lambda=0.45)$ at $\tan \beta=2$. As $\tan \beta$ increases, the dependence of top squark masses on $\lambda$, as well as $v_{s}$ weakens, therefore these elements of the mass-squared matrix become more sensitive to the choice of these parameters. Hence, for $\tan \beta=10$ (right panel of Fig. 6), one observes a slow variation with $\varphi$, and quite large splitting between the tree and loop levels as compared to $\lambda=0.12$ case (see Fig. 2, right panel). Similar analysis can be performed for $v_{s} \gtrsim 2 v$, and $\lambda=0.45$, in which case one observes a weak dependence of $\varphi$ on $m_{h_{5}}$ at both $\tan \beta=2$ and $\tan \beta=10$ regimes (see Fig. 7).
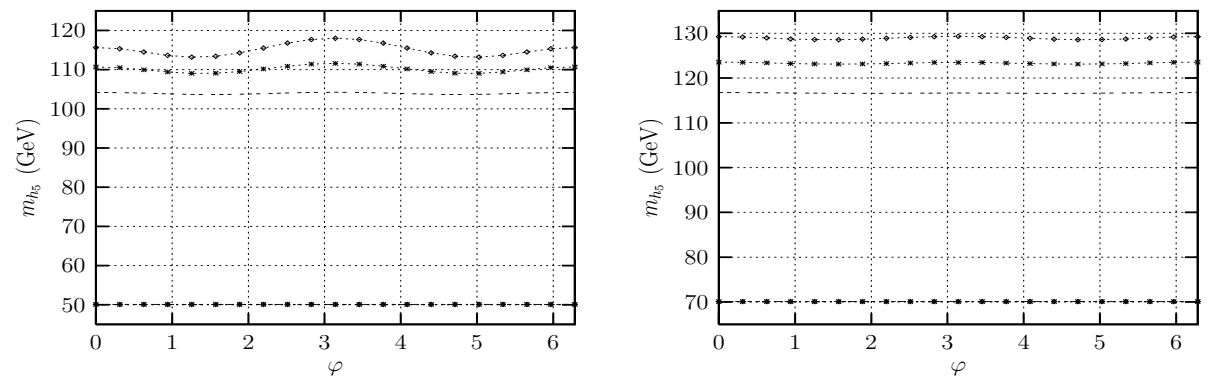

Fig. 7. The dependence of $m_{h_{5}}$ on $\varphi$ for $v_{s}=525 \mathrm{GeV}$, and $\lambda=0.45$, for selected values of $A_{t}$, when $\tan \beta=2$ (left panel), and $\tan \beta=10$ (right panel). Here, the bottom, middle and the top curves are for $A_{t}=v_{s}=525 \mathrm{GeV}, A_{t}=1050 \mathrm{GeV}$, and $A_{t}=1400 \mathrm{GeV}$, respectively, whereas the lowest curve is for the tree-level.

One notes from the left panel Fig. 7 that the increase in $v_{s}$ affects the radiative corrections, which causes quite large splittings between the tree and the loop levels, at $\tan \beta=2$. On the other hand, it is seen from the right panel that when $\tan \beta=$ 10, the variation of $\varphi$ is much more flat, as compared to right panel of Fig. 6.

In Fig. 8, we show the dependence of $\rho_{3}$ and $\rho_{5}$ on $\varphi$, for $\lambda=0.45$, and $v_{s}=v$, when $\tan \beta=2$ (left panel) and $\tan \beta=10$ (right panel). From the left panel it is seen that when $A_{t}=1050 \mathrm{GeV}\left(A_{t}=1400 \mathrm{GeV}\right)$, the $\varphi$ dependence of $\rho_{3}$ (the upper curves) and $\rho_{5}$ (the lower curves) is stronger as compared to $A_{t}=v_{s}$ (the top curve 

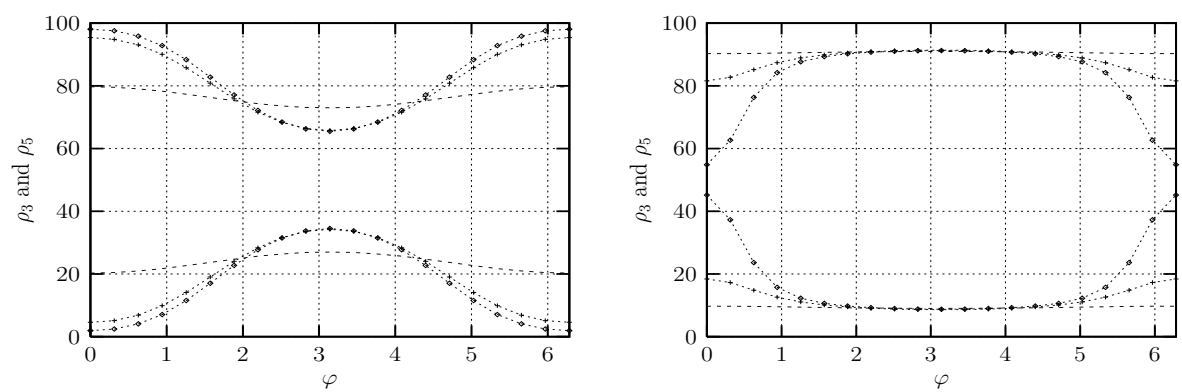

Fig. 8. The dependence of $\rho_{3}$ and $\rho_{5}$ on $\varphi$ for $v_{s}=\mathrm{v}$, and $\lambda=0.45$, for selected values of $A_{t}$, when $\tan \beta=2$ (left panel), and $\tan \beta=10$ (right panel). The upper (lower) curves are for $\rho_{3}\left(\rho_{5}\right)$ in the left panel, whereas they are for $\rho_{5}\left(\rho_{3}\right)$ in the right panel. Here, $A_{t}=v_{s}=175 \mathrm{GeV}$, $A_{t}=1050 \mathrm{GeV}, A_{t}=1400 \mathrm{GeV}$, from top to bottom for $\rho_{3}\left(\rho_{5}\right)$, with respect to the mid-point.

with respect to the mid-point). It is seen that $\operatorname{as} \tan \beta$ increases, the contributions of the CP odd components change. For instance, at $\tan \beta=2$ (left panel), and $A_{t}=8 v_{s} \rho_{3}$ starts from $\% 98$ at $\varphi=0$ and falls to $\% 65$ at $\varphi=\pi$ ( $\rho_{5}$ varies from $\% 2$ to $\% 37$ in this interval). In passing to $\tan \beta=10$ regime (right panel) $h_{5}$ is seen to gain non-negligible $\rho_{5}$ composition. For instance at $A_{t}=1400 \mathrm{GeV}, \rho_{5}$ starts with $\% 50$ at $\varphi=0$, and rises to $\% 90$ at $\varphi=\pi$. Similar to the previous observations, it can be observed that when $A_{t}$ and $v_{s}$ are of comparible size, the strengths of the $\mathrm{CP}$ violation mixing is weak and the variations of $\rho_{3}$ and $\rho_{5}$ with $\varphi$ are quite slow. As the figure suggests, the higher $A_{t}$ the larger the $\mathrm{CP}$ violating mixings between the CP odd components. One notes that similar analysis can be carried out for the higher values of $v_{s}$. It can be seen that, as $v_{s}$ increases, $\rho_{5}$ component of $h_{3}$ increases, and this increase in the $\rho_{5}$ component is compensated by $\rho_{3}$.

\subsection{The Case of Complex $\lambda$}

In the second part of the analysis, we take $\lambda$ as a complex parameter, with the phase $\varphi_{\lambda}$. Then, we let all the other soft phases in the theory of being zero $\left(\varphi_{A_{t}}=\right.$ $\left.\varphi_{A_{b}}=\varphi_{A_{e}}=\varphi_{1}=\varphi_{2}=0\right)$. to explore the dependence of $\varphi_{\lambda}$ on various parameters.
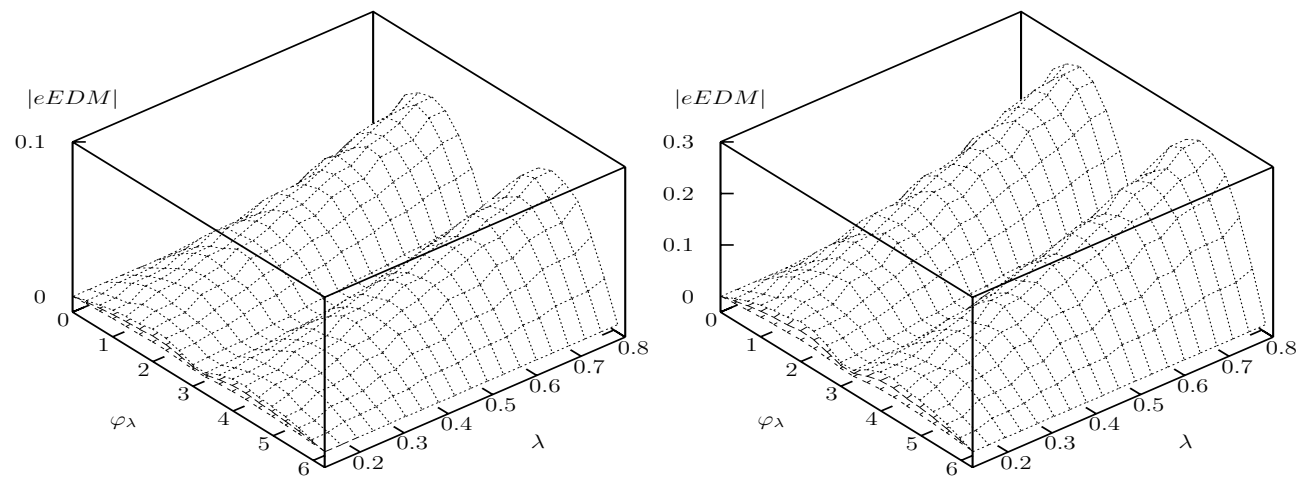

Fig. 9. The dependence of $|e E D M|$ on $\varphi_{\lambda}$ and $\lambda$ for selected values of the vacuum expectation value of the singlet when $\tan \beta=2$ (left panel) and $\tan \beta=10$ (right panel). 
In Fig. 9, we show the dependence of $|e E D M|$ on $\varphi_{\lambda}$ and $\lambda$ for selected values of $v_{s}$ when $\tan \beta=2$ (left panel) and $\tan \beta=10$ (right panel). Similar to the former analysis, we fix $k=0.63$, and consider all values of $\lambda$ in the $0.12 \lesssim \lambda \lesssim 0.82$, interval, as $\varphi_{\lambda}$ changes from 0 to $2 \pi$. In both panels of the Figure, the top surface corresponds to $v_{s}=4 v$, whereas the bottom represents $v_{s}=v$. A comparative look at both panels of Fig. 9 suggests that the present bounds of $|e E D M|$ is satisfied in all the parameter domain at both $\tan \beta$ regimes, when $\varphi_{\lambda}$ changes in its full range and $\lambda$ from 0.12 to 0.82 , provided that the gaugino masses are of $\mathcal{O}(\mathrm{TeV})$.

In the following, we will perform a similar analysis that we have carried out for the real $\lambda$ case. To understand the effects of $\varphi_{\lambda}$ on $m_{h_{5}}$, as well as $\rho_{3}$ and $\rho_{5}$, we first start with a low value of $\lambda$, that is we set $\lambda=0.12$, and again consider two specific values of $v_{s}$, namely $v_{s}=175 \mathrm{GeV}$, and $v_{s}=525 \mathrm{GeV}$. We show the dependence of $m_{h_{5}}$ (at tree and at one-loop levels) on $\varphi_{\lambda}$, in Fig. 10 (when $v_{s}=v$ ), and Fig. $11\left(v_{s}=3 v\right)$ at $\tan \beta=2$ (left panels) and $\tan \beta=10$ (right panels).
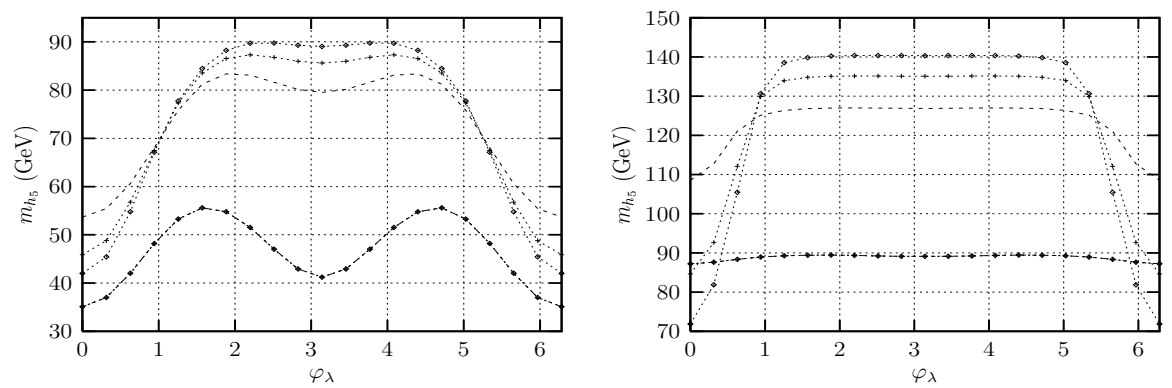

Fig. 10. The dependence of $m_{h_{5}}$ on $\varphi_{\lambda}$, for selected values of $A_{t}$, at $\tan \beta=2$ (left panel) and $\tan \beta=10$ (right panel), when $v_{s}=175 \mathrm{GeV}, \mathrm{v}$ and $\lambda=0.12$. Here, the bottom, middle and the top curves (with respect to the mid-point) are for $A_{t}=v_{s}=175 \mathrm{GeV}, A_{t}=1050 \mathrm{GeV}$, and $A_{t}=1400 \mathrm{GeV}$, respectively, whereas the lowest curve is for the tree-level.

As the left panel of Fig. 10 indicates $m_{h_{5}}$ is quite sensitive to the variations of $\varphi_{\lambda}$ when $v_{s}=v$, and $\tan \beta=2$. For $\tan \beta=10$ regime, it is seen that the variation of $\varphi_{\lambda}$ with $m_{h_{5}}$ is slower at both tree and one-loop levels, particularly in the $1 \lesssim \varphi_{\lambda} \lesssim 5$ interval.
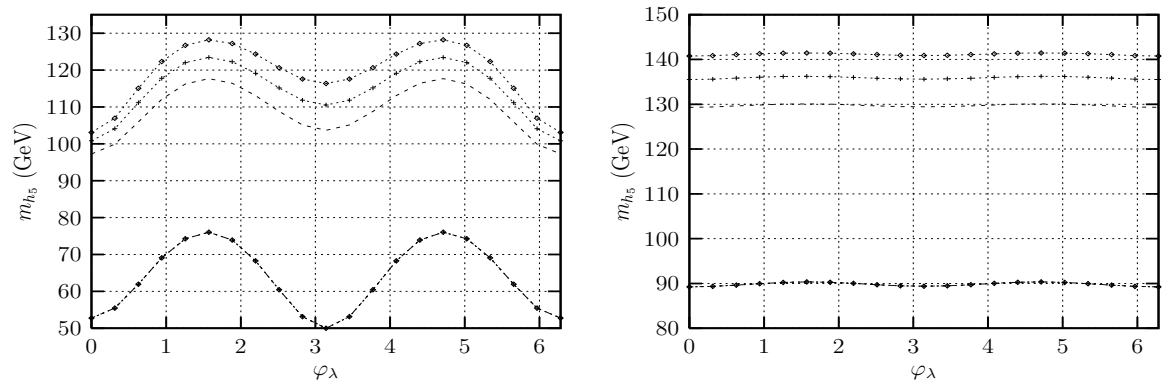

Fig. 11. The dependence of $m_{h_{5}}$ on $\varphi_{\lambda}$, for selected values of $A_{t}$, at $\tan \beta=2$ (left panel) and $\tan \beta=10$ (right panel), when $v_{s}=525 \mathrm{GeV}$ and $\lambda=0.12$. Here, the bottom, the middle and the top curves, are for $A_{t}=v_{s}=525 \mathrm{GeV}, A_{t}=1050 \mathrm{GeV}$, and $A_{t}=1400 \mathrm{GeV}$, respectively, whereas the lowest curve is for the tree level.

In passing to $v_{s}=3 v$ case (see Fig. 11), although $m_{h_{5}}$ remains quite flat for 
$\tan \beta=10$ (right panel), one obtains a relatively fast variation for $\tan \beta=2$. It is seen that from the left panel of Fig. 11 that the peak value of $m_{h_{5}}$ at the oneloop level, as well as the tree level, is shifted through the maximal $\mathrm{CP}$ violation point at $\tan \beta=2$. A comparative analysis of Figs. 10 and 11 suggest that $m_{h_{5}}$ is quite sensitive to the variations in $\varphi_{\lambda}$ for $\lambda=0.12$, when $v_{s}=v$ and $v_{s}=3 v$ at $\tan \beta=2$ (left panels) regime. Such kind of sensitivity weakens at $\tan \beta=10$ (right panels) for $v_{s} \gtrsim 2 v$.

We show the dependence of CP-odd components $\left(\rho_{3}\right.$ and $\left.\rho_{5}\right)$, of $h_{5}$ on $\varphi_{\lambda}$, for $\lambda=0.12$, and $v_{s}=v$ in Fig. 12 , and $v_{s}=3 v$ in Fig. 13, respectively, when $\tan \beta=2$ (left panels) and $\tan \beta=10$ (right panels). As can be observed from the
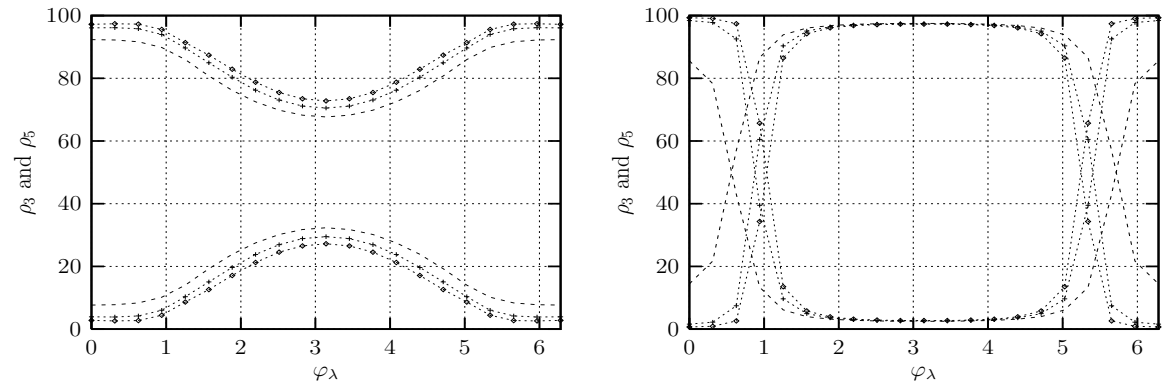

Fig. 12. The dependence of $\rho_{3}$ (the upper curves) and $\rho_{5}$ (the lower curves) on $\varphi_{\lambda}$ for $v_{s}=$ $175 \mathrm{GeV}$, and $\lambda=0.12$, when $\tan \beta=2$ (left panel), and $\tan \beta=10$ (right panel). Here, $A_{t}=v_{s}=$ $175 \mathrm{GeV}, A_{t}=1050 \mathrm{GeV}, A_{t}=1400 \mathrm{GeV}$, from bottom to top for $\rho_{3}$, and from top to bottom for $\rho_{5}$, with respect to the mid-point.

left panel of Fig. 12, when $\tan \beta=2, \rho_{3}$ component of $h_{5}$ on the average ranges from $\% 98$ to $\% 90$, as $A_{t}$ changes from $1400 \mathrm{GeV}$ to $175 \mathrm{GeV}$ (from top to bottom for the upper lines) at $\varphi_{\lambda}=0$. In passing to $\tan \beta=10$ regime, it is seen that $h_{5}$ has nearly $\% 100 \rho_{3}$ component at $\varphi_{\lambda}=0$, as $A_{t}$ changes from $1400 \mathrm{GeV}$ to $1050 \mathrm{GeV}$ (the first and the second curve from the top of the upper lines representing $\rho_{3}$ ). Moreover, $h_{5}$ is seen to gain non-negligible $\rho_{5}$ component in such a way that $\rho_{3}$ and $\rho_{5}$ components are strongly mixed up in this regime (right panel).
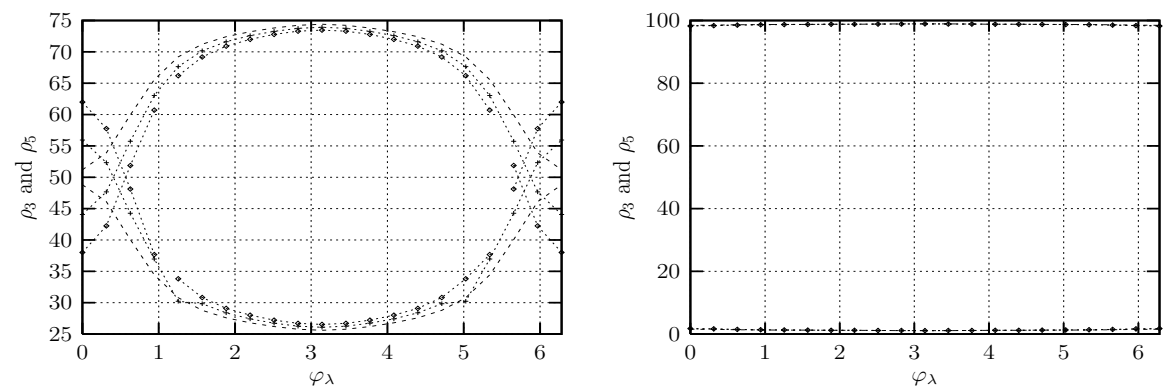

Fig. 13. The dependence of $\rho_{3}$ (the lower curves) and $\rho_{5}$ (the upper curves) on $\varphi_{\lambda}$ for $v_{s}=$ $525 \mathrm{GeV}$, and $\lambda=0.12$, when $\tan \beta=2$ (left panel), and $\tan \beta=10$ (right panel). Here, $A_{t}=v_{s}=$ $525 \mathrm{GeV}, A_{t}=1050 \mathrm{GeV}, A_{t}=1400 \mathrm{GeV}$, from bottom to top for $\rho_{3}$, and from top to bottom for $\rho_{5}$, with respect to the mid-point.

On the other, considering the higher values of $v_{s}$ (see Fig. 13), one notes that the $\rho_{5}$ component of $h_{5}$ increases, and this increase in the $\rho_{5}$ is compensated by 
$\rho_{3}$ when $\tan \beta=2$ (left panel). For $\tan \beta=10$ (right panel) regime, it can be observed that $\rho_{5}$ component of $h_{5}$ has a slow variation in the vicinity of $\% 100$ line, whereas $\rho_{3}$ remains around $\% 0$ line, for each value of $A_{t}$, changing from $525 \mathrm{GeV}$ to $A_{t}=1400 \mathrm{GeV}$.

In the last part of our analysis, we fix $\lambda=0.45$, and carry out the similar analysis for $v_{s}=v$ and $v_{s}=3 v$. We show the dependence of $m_{h_{5}}$ (at tree and at one-loop levels) on $\varphi_{\lambda}$ for $v_{s}=v$ in Fig. 14, and $v_{s}=3 v$ in Fig. 15, when $\tan \beta=2$ (left panels) and $\tan \beta=10$ (right panels). In Figs. 14 and $15, A_{t}=1400 \mathrm{GeV}$ (the top curve with respect to the mid-point), $A_{t}=1050 \mathrm{GeV}$ (the second curve below the top curve), whereas the third curve below the top curve is for $A_{t}=v_{s}=175 \mathrm{GeV}$ in Fig. 14, and $A_{t}=v_{s}=525 \mathrm{GeV}$ in Fig. 15. In both Figures, the lowest curve represents $m_{h_{5}}$ at the tree-level.
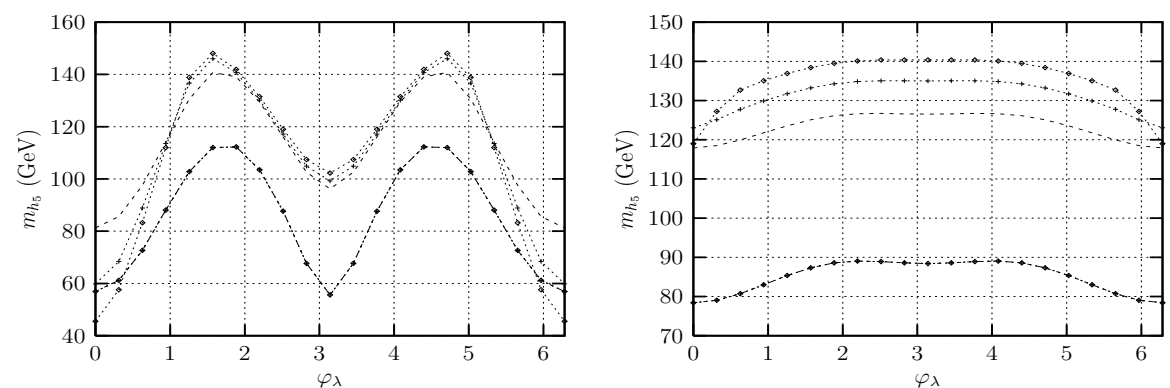

Fig. 14. The dependence of $m_{h_{5}}$ on $\varphi_{\lambda}$ for $v_{s}=\mathrm{v}$, and $\lambda=0.45$, for selected values of $A_{t}$, when $\tan \beta=2$ (left panel), and $\tan \beta=10$ (right panel). Here, the bottom, the middle and the top curves, with respect to the mid-point, are for $A_{t}=v_{s}=175 \mathrm{GeV}, A_{t}=1050 \mathrm{GeV}$, and $A_{t}=1400 \mathrm{GeV}$ values, whereas the lowest curve is for the tree level.
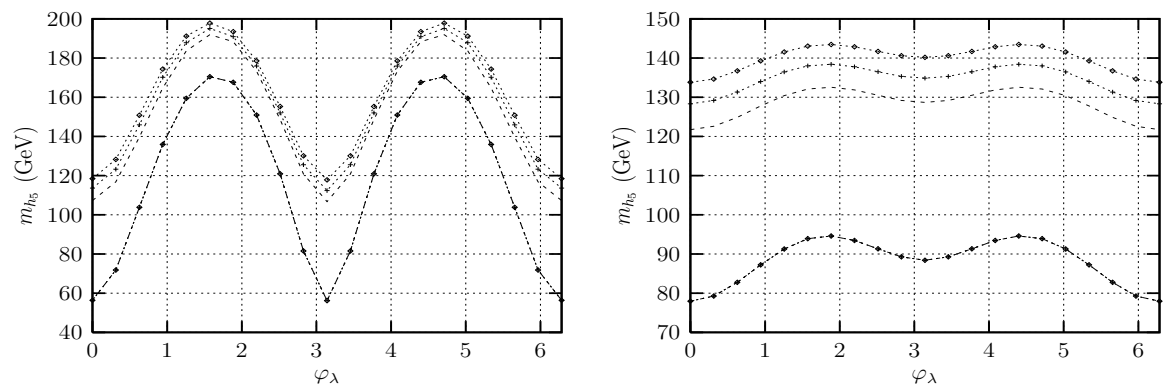

Fig. 15. The dependence of $m_{h_{5}}$ on $\varphi_{\lambda}$ for $v_{s}=3 \mathrm{v}$, and $\lambda=0.45$, for selected values of $A_{t}$, when $\tan \beta=2$ (left panel), and $\tan \beta=10$ (right panel). Here, the bottom, the middle and the top curves, with respect to the mid-point, are for $A_{t}=v_{s}=525 \mathrm{GeV}, A_{t}=1050 \mathrm{GeV}$, and $A_{t}=1400 \mathrm{GeV}$ values, whereas the lowest curve is for the tree level.

One notes from the left panels of Figs. 14 and 15 that as $\lambda$ increases $(\lambda=0.45)$, one obtains a quite fast variation of $m_{h_{5}}$ with $\varphi_{\lambda}$ both at the tree and loop levels, particularly in the low-tan $\beta$ regime. The increase in $\lambda$, as well as $v_{s}$ affects the spectrum of $h_{5}$ in the sense that the shifting of the peak value of $m_{h_{5}}$ (at the tree and the loop levels) towards the maximal $\mathrm{CP}$ violation point is sharper as compared to the $\lambda=0.12$ case (see Fig. 11). Although the sensitivity much more weakens in the high $\tan \beta$ regime, such kind of shifting effect of $m_{h_{5}}$ can also be observed from the right panel of Fig. 15. 
Finally, in Fig. 16, we show the dependence of CP-odd components $\left(\rho_{3}\right.$ and $\left.\rho_{5}\right)$ of $h_{3}$ on $\varphi_{\lambda}$, for $\lambda=0.45$, and $v_{s}=v$ at $\tan \beta=2$ (left panel) and $\tan \beta=10$ (right panel). As can be observed from Fig. 16, the increase in $\lambda$ affects the CP odd components in such a way that $\rho_{3}$ and $\rho_{5}$ mix significantly in the $\tan \beta=2$ regime. In passing to $\tan \beta=10$ regime, it is seen that such mixings weaken, however $h_{5}$ is seen to gain non-negligible $\rho_{5}$ component as $A_{t}$ increases. On the other hand, as $v_{s}$ increases, it can be seen that $\rho_{3}$ and $\rho_{5}$ have similar $\varphi_{\lambda}$ dependencies for all values of $A_{t}$, which essentially follows from the dominance of the $A_{t} \lambda v_{s}$ term.
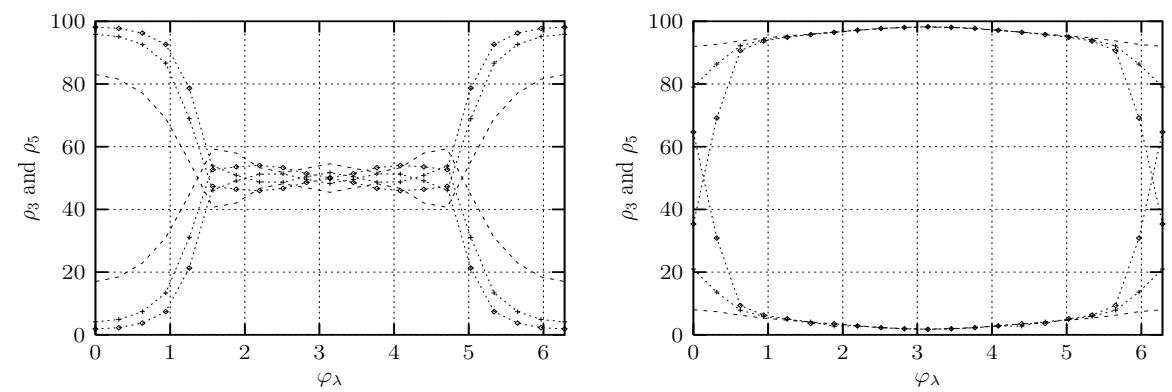

Fig. 16. The dependence of $\rho_{3}$ and $\rho_{5}$ on $\varphi_{\lambda}$ for $v_{s}=\mathrm{v}$, and $\lambda=0.45$, for selected values of $A_{t}$, when $\tan \beta=2$ (left panel), and $\tan \beta=10$ (right panel). The upper (lower) curves are for $\rho_{3}\left(\rho_{5}\right)$ in the left panel, whereas they are for $\rho_{5}\left(\rho_{3}\right)$ in the right panel. Here, $A_{t}=v_{s}=175 \mathrm{GeV}$, $A_{t}=1050 \mathrm{GeV}, A_{t}=1400 \mathrm{GeV}$, from top to bottom for $\rho_{3}\left(\rho_{5}\right)$, with respect to the mid-point.

Before concluding, we would like to close this section with a brief discussion of the measurement of CP violating effects at colliders: Recently, the authors of Ref. [44] have presented a general formalism, the resonant $\mathrm{CP}$ violation, for analyzing the $\mathrm{CP}$ violating phenomena in the MSSM at high-energy colliders. Their formalism, which is developed from Ref. [45], can be applied to models with an extended $\mathrm{CP}$ violating Higgs sector, including the MSSM with radiative Higgs-sector CP violation. Resonant CP violation has not been studied in the NMSSM yet. The measurement of resonant $\mathrm{CP}$ violation effects can provide a useful tool for the determination of the CP odd compositions of the Higgs bosons in the NMSSM.

\section{Conclusion}

We study the explicit CP violation of the Higgs sector in the next-to-minimal supersymmetric model with a gauge singlet Higgs field. Our general discussion followed by the numerical estimates for various parameter planes show that:

(i)The eEDM lies around the present experimental upper limits, provided that the gaugino masses are of $\mathcal{O}(\mathrm{TeV})$.

(ii) When $\lambda$ is real, and all the other phases in the theory are complex, $m_{h_{5}}$ is quite sensitive to $\varphi$, particularly at small $\lambda$ and $v_{s}\left(\lambda=0.12\right.$, and $\left.v_{s}=v\right)$. However as $v_{s}$ and $\tan \beta$ increases (for instance, $\lambda=0.12, v_{s}=3 v$ and $\tan \beta=10$ ), the radiative corrections which are sensitive to variations in $\varphi$ are suppressed. Indeed, $m_{h_{5}}$ nearly remains constant for $\tan \beta=10$ at $v_{s}=3 v$. Considering the $\rho_{3}$ and $\rho_{5}$ components of $h_{5}$ at small $\lambda$ and $v_{s}\left(\lambda=0.12\right.$, and $\left.v_{s}=v\right)$, as $A_{t}$ increases, the $\rho_{3}$ component of $h_{5}$ decreases, whereas its $\rho_{5}$ component increases. However, this increase in $\rho_{5}$ can not be larger than $\% 20$, at $\tan \beta=2$, even for $A_{t}=1400 \mathrm{GeV}$. In passing to $\tan \beta=10$ regime, it is seen that the $\rho_{3}$ component of $h_{5}$ tend to decrease more rapidly, whereas this decrease is compensated by the increase in $\rho_{5}$, in the sense that the two components mix around $\varphi=\pi$, when $\lambda=0.12$. On the other hand, for higher values of $v_{s}\left(v_{s}=3 v\right)$, it is seen that the variation of the CP odd components is quite slow, as compared to the former case. 
(iii) When $\lambda$ is complex, and all the other CP violating parameters in the theory are chosen to be real, $m_{h_{5}}$ grows significantly as compared to the former case. It is observed that $\varphi_{\lambda}$ strongly affect the the tree and one-loop Higgs masses. Clearly, $\varphi_{\lambda}$ is not only a physical phase which forms the source of $\mathrm{CP}$ violation at the tree level, but it also affects the one-loop radiative corrections via $\varphi_{\lambda t}$. Therefore, even if all the other phases are set equal to zero. the effects of $\varphi_{\lambda}$ can be seen on the mass and the CP components of $h_{5}$. The CP violating effects are particularly enhanced, as $v_{s}$ increases.

(iv)Being the most economic extension of the MSSM, the next-to-minimal supersymmetric model not only leads us to a wealth of CP violation opportunities, but it also offers a rich phenomenology for future colliders.

1. P. G. Harris et al., Phys. Rev. Lett. 82, 904 (1999).

2. R. D. Peccei and H. R. Quinn, Phys. Rev. Lett. 38, 1440 (1977).

3. S. Weinberg, Phys. Rev. Lett. 40, 223 (1978); F. Wilczek, Phys. Rev. Lett. 40, 279 (1978)

4. J. E. Kim, Phys. Rev. Lett. 43, 103 (1979); M. A. Shifman, A. I. Vainshtein and V. I. Zakharov, Nucl. Phys. B166, 493 (1980); M. Dine, W. Fischler and M. Srednicki, Phys. Lett. B104, 199 (1981).

5. S. Dimopoulos and S. Thomas, Nucl. Phys. B465, 23 (1996) [arXiv:hep-ph/9510220].

6. D. A. Demir, Phys. Rev. D 62, 075003 (2000) [arXiv:hep-ph/9911435].

7. M. Dugan, B. Grinstein and L. Hall, Nucl. Phys. B255, 413 (1985).

8. T. Ibrahim and P. Nath, Phys. Rev. D 58, 111301 (1998) [Erratum-ibid. D 60, 099902 (1999)] [arXiv:hep-ph/9807501]; Phys. Rev. D 61, 093004 (2000) [arXiv:hepph/9910553]; T. Ibrahim, Phys. Rev. D 64, 035009 (2001) [arXiv:hep-ph/0102218].

9. D. A. Demir, M. Pospelov and A. Ritz, Phys. Rev. D 67, 015007 (2003) [arXiv:hep$\mathrm{ph} / 0208257]$.

10. S. Abel, S. Khalil and O. Lebedev, Nucl. Phys. B 606, 151 (2001) [arXiv:hep$\mathrm{ph} / 0103320]$.

11. A. Pilaftsis, Nucl. Phys. B 644, 263 (2002) [arXiv:hep-ph/0207277].

12. O. Lebedev and M. Pospelov, Phys. Rev. Lett. 89, 101801 (2002) [arXiv:hep$\mathrm{ph} / 0204359]$.

13. M. Boz and N. Karagoz, Mod. Phys. Lett. A 18, 1601 (2003).

14. A. Pilaftsis, Phys. Lett. B 435, 88 (1998) [arXiv:hep-ph/9805373]; Phys. Rev. D 58, 096010 (1998) [arXiv:hep-ph/9803297]; A. Pilaftsis and C. E. Wagner, Nucl. Phys. B 553, 3 (1999) [arXiv:hep-ph/9902371]; D. A. Demir, Phys. Rev. D 60, 055006 (1999) [arXiv:hep-ph/9901389]; Nucl. Phys. Proc. Suppl. 81, 224 (2000) [arXiv:hepph/9907279]; M. Carena, J. R. Ellis, A. Pilaftsis and C. E. Wagner, Nucl. Phys. B 586, 92 (2000) [arXiv:hep-ph/0003180]; S. Y. Choi, M. Drees and J. S. Lee, Phys. Lett. B 481, 57 (2000) [arXiv:hep-ph/0002287]; S. W. Ham, S. K. Oh, E. J. Yoo and H. K. Lee, J. Phys. G 27, 1 (2001); T. Ibrahim and P. Nath, Phys. Rev. D 63, 035009 (2001) [arXiv:hep-ph/0008237].

15. M. Boz, Mod. Phys. Lett. A 17, 215 (2002) [arXiv:hep-ph/0008052]; J. Phys. G 28, 2377 (2002) [arXiv:hep-ph/0207050]; Mod. Phys. Lett. A 19, 433 (2004) [arXiv:hepph/0311248].

16. M. Boz and N. K. Pak, Phys. Rev. D 65, 075014 (2002); A. Dedes and A. Pilaftsis, Phys. Rev. D 67, 015012 (2003) [arXiv:hep-ph/0209306]; D. A. Demir, Phys. Lett. B 571, 193 (2003) [arXiv:hep-ph/0303249];

17. D. A. Demir and K. A. Olive, Phys. Rev. D 65, 034007 (2002) [arXiv:hep-ph/0107329]; M. Boz and N. K. Pak, Phys. Lett. B 531, 119 (2002) [arXiv:hep-ph/0201199].

18. J. R. Espinosa and M. Quiros, Phys. Lett. B 279, 92 (1992); Phys. Lett. B 302, 51 (1993) [arXiv:hep-ph/9212305]; G. L. Kane, C. F. Kolda and J. D. Wells, Phys. Rev. 
Lett. 70, 2686 (1993) [arXiv:hep-ph/9210242]; T. Elliott, S. F. King and P. L. White, Phys. Lett. B 305, 71 (1993) [arXiv:hep-ph/9302202]; F. Franke, H. Fraas and A. Bartl, Phys. Lett. B 336, 415 (1994) [arXiv:hep-ph/9408217]; U. Ellwanger, M. Rausch de Traubenberg and C. A. Savoy, Z. Phys. C 67, 665 (1995) [arXiv:hep-ph/9502206]; D. A. Demir, arXiv:hep-ph/9902468;

19. U. Ellwanger, J. F. Gunion and C. Hugonie, arXiv:hep-ph/0503203.

20. D. J. Miller and S. Moretti, arXiv:hep-ph/0403137.

21. The LEP Higgs Working Group for Higgs boson searches, arXiv:hep-ex/0107029; arXiv:hep-ex/0107030; ALEPH,DELPHI,L3 and OPAL Collaborations, The LEP Higgs Working Group for Higgs Boson Searches, LHWG Note/2001-04 (2001), LHWG Note/2002-01 (2002), http://www.cern.ch/LEPHIGGS/www/Welcome.html.

22. M. Bastero-Gil, C. Hugonie, S. F. King, D. P. Roy and S. Vempati, Phys. Lett. B 489, 359 (2000) [arXiv:hep-ph/0006198].

23. R. Dermisek and J. F. Gunion, arXiv:hep-ph/0502105.

24. N. Maekawa, Phys. Lett. B 282, 387 (1992); A. Pomarol, Phys. Lett. B 287, 331 (1992). [hep-ph/9205247].

25. G. C. Branco, F. Kruger, J. C. Romao and A. M. Teixeira, JHEP 0107, 027 (2001) [arXiv:hep-ph/0012318].

26. A. T. Davies, C. D. Froggatt and A. Usai, Phys. Lett. B 517, 375 (2001) [arXiv:hep$\mathrm{ph} / 0105266]$.

27. J. C. Romao, Phys. Lett. B 173, 309 (1986).

28. N. Haba, M. Matsuda and M. Tanimoto, Phys. Rev. D 54, 6928 (1996) [arXiv:hepph/9512421]; S. W. Ham, S. K. Oh and H. S. Song, Phys. Rev. D 61, 055010 (2000) [arXiv:hep-ph/9910461].

29. N. Haba, M. Matsuda and M. Tanimoto, Prog. Theor. Phys. Suppl. 123, 265 (1996).

30. M. Matsuda and M. Tanimoto, Phys. Rev. D 52, 3100 (1995) [arXiv:hep-ph/9504260];

31. U. Ellwanger, Phys. Lett. B 303, 271 (1993) [arXiv:hep-ph/9302224]; T. Elliott, S. F. King and P. L. White, Phys. Lett. B 314, 56 (1993) [arXiv:hep-ph/9305282]; Phys. Rev. D 49, 2435 (1994) [arXiv:hep-ph/9308309]; P. N. Pandita, Phys. Lett. B 318, 338 (1993); Z. Phys. C 59, 575 (1993).

32. S. W. Ham, J. Kim, S. K. Oh and D. Son, Phys. Rev. D 64, 035007 (2001) [arXiv:hepph/0104144];

33. S. W. Ham, S. K. Oh and D. Son, Phys. Rev. D 65, 075004 (2002) [arXiv:hep$\mathrm{ph} / 0110052]$

34. S. W. Ham, Y. S. Jeong and S. K. Oh, arXiv:hep-ph/0308264.

35. C. Panagiotakopoulos and A. Pilaftsis, Phys. Rev. D 63, 055003 (2001) [arXiv:hep$\mathrm{ph} / 0008268]$.

36. S. W. Ham, S. K. OH, E. J. Yoo, C. M. Kim and D. Son, arXiv:hep-ph/0406070.

37. J. R. Ellis, J. F. Gunion, H. E. Haber, L. Roszkowski and F. Zwirner, Phys. Rev. D 39, 844 (1989).

38. D. J. Miller, R. Nevzorov and P. M. Zerwas, Nucl. Phys. B 681, 3 (2004) [arXiv:hep$\mathrm{ph} / 0304049]$.

39. T. Ibrahim and P. Nath, Phys. Lett. B 418, 98 (1998) [arXiv:hep-ph/9707409]; Phys. Rev. D 57, 478 (1998) [Erratum-ibid. D 58, 019901 (1998 ERRAT,D60,079903.1999 ERRAT,D60,119901.1999)] [arXiv:hep-ph/9708456].

40. D. Chang, W. Y. Keung and A. Pilaftsis, Phys. Rev. Lett. 82, 900 (1999) [Erratumibid. 83, 3972 (1999)] [arXiv:hep-ph/9811202]; A. Pilaftsis, Phys. Lett. B 471, 174 (1999) [arXiv:hep-ph/9909485]; D. Chang, W. F. Chang and W. Y. Keung, Phys. Lett. B 478, 239 (2000) [arXiv:hep-ph/9910465].

41. E. D. Commins, S. B. Ross, D. DeMille and B. C. Regan, Phys. Rev. A 50, 2960 (1994). 
42. K. Abdullah, C. Carlberg, E. D. Commins, H. Gould and S. B. Ross, Phys. Rev. Lett. 65, 2347 (1990).

43. D. A. Demir, Nucl. Phys. Proc. Suppl. 101, 431 (2001).

44. J. R. Ellis, J. S. Lee and A. Pilaftsis, Phys. Rev. D 70, 075010 (2004) [arXiv:hepph/0404167]; Nucl. Phys. B 718, 247 (2005) [arXiv:hep-ph/0411379]; arXiv:hepph/0507046.

45. A. Pilaftsis, Nucl. Phys. B 504, 61 (1997) [arXiv:hep-ph/9702393]. 